\title{
Toward complete oral cavity cancer resection using a handheld diffuse reflectance spectroscopy probe
}

Susan G. Brouwer de Koning

Elisabeth J. M. Baltussen

M. Baris Karakullukcu

Behdad Dashtbozorg

Laura A. Smit

Richard Dirven

Benno H. W. Hendriks

Henricus J. C. M. Sterenborg

Theo J. M. Ruers 


\title{
Toward complete oral cavity cancer resection using a handheld diffuse reflectance spectroscopy probe
}

\author{
Susan G. Brouwer de Koning, ${ }^{a, *}$ Elisabeth J. M. Baltussen, ${ }^{a}$ M. Baris Karakullukcu, ${ }^{a}$ Behdad Dashtbozorg, ${ }^{a}$ \\ Laura A. Smit, ${ }^{\mathrm{b}}$ Richard Dirven, ${ }^{\mathrm{a}}$ Benno H. W. Hendriks, ${ }^{\mathrm{c}, \mathrm{d}}$ Henricus J. C. M. Sterenborg, ${ }^{\mathrm{a}, \mathrm{e}}$ and \\ Theo J. M. Ruers ${ }^{a, f}$ \\ aNetherlands Cancer Institute, Antoni van Leeuwenhoek, Department of Surgery, Amsterdam, The Netherlands \\ ${ }^{b}$ Netherlands Cancer Institute, Antoni van Leeuwenhoek, Department of Pathology, Amsterdam, The Netherlands \\ 'Philips Research, Department of In-body Systems, Eindhoven, The Netherlands \\ ${ }^{\mathrm{d} D e l f t}$ University of Technology, Department of Biomechanical Engineering, Delft, The Netherlands \\ ${ }^{\mathrm{e}}$ Academic Medical Centre, Department of Biomedical Engineering and Physics, Amsterdam, The Netherlands \\ fUniversity of Twente, MIRA Institute, Enschede, The Netherlands
}

\begin{abstract}
This ex-vivo study evaluates the feasibility of diffuse reflectance spectroscopy (DRS) for discriminating tumor from healthy tissue, with the aim to develop a technology that can assess resection margins for the presence of tumor cells during oral cavity cancer surgery. Diffuse reflectance spectra were acquired on fresh surgical specimens from 28 patients with oral cavity squamous cell carcinoma. The spectra (400 to $1600 \mathrm{~nm}$ ) were detected after illuminating tissue with a source fiber at 0.3-, 0.7-, 1.0-, and 2.0-mm distances from a detection fiber, obtaining spectral information from different sampling depths. The spectra were correlated with histopathology. A total of 76 spectra were obtained from tumor tissue and 110 spectra from healthy muscle tissue. The first- and second-order derivatives of the spectra were calculated and a classification algorithm was developed using fivefold cross validation with a linear support vector machine. The best results were obtained by the reflectance measured with a 1-mm source-detector distance (sensitivity, specificity, and accuracy are $89 \%, 82 \%$, and $86 \%$, respectively). DRS can accurately discriminate tumor from healthy tissue in an ex-vivo setting using a 1-mm source-detector distance. Accurate validation methods are warranted for larger sampling depths to allow for guidance during oral cavity cancer excision. @ 2018 Society of Photo-Optical Instrumentation Engineers (SPIE) [DOI: 10.1117/1.JBO.23.12.121611]
\end{abstract}

Keywords: resection margin assessment; diffuse reflectance spectroscopy; oral cavity cancer; tissue recognition; machine learning; linear support vector machine.

Paper 180424SSR received Jun. 30, 2018; accepted for publication Sep. 26, 2018; published online Oct. 19, 2018.

\section{Introduction}

Patients with early stage oral cavity squamous cell carcinoma are generally treated with surgery. Surgeons aim to remove the tumor with a margin of healthy tissue, to ensure optimal local control and prognosis.

The extent of the margin is a trade-off between removing enough tissue to ensure clear margins and sparing as much healthy tissue as possible for good functional outcome. During surgery, information about the extent of the tumor is limited to the surgeon's palpation of the tumor and preoperative radiological assessment with magnetic resonance (MR) and/ or ultrasound (US) imaging. Other than palpation, there is no real-time feedback about tumor borders. In general, the deep resection plane is the most challenging location to achieve well clear margins. Hence, in up to $30 \%$ to $85 \%$ of the patients, the tumor is removed with an involved or close margin of healthy tissue (i.e., an involved margin, in which tumor cells are present at the resection surface, or a close margin, in which tumor cells are found within $5 \mathrm{~mm}$ from the resection surface). ${ }^{1}$ The majority of these patients need adjuvant treatment with additional surgery, radiotherapy, or sometimes chemo radiation. To reduce the number of patients needing adjuvant treatment, there is an urgent need for a technology that can

*Address all correspondence to Susan G. Brouwer de Koning, E-mail: s.brouwerdekoning@nki.nl provide real-time feedback on the presence of tumor cells at the resection margins.

In diffuse reflectance spectroscopy (DRS), diffusely reflected light is measured after illuminating the tissue with a broadband white light source using fiber optics. The reflectance spectrum contains information about the absorption and scattering properties of the illuminated tissue, representing an "optical fingerprint" of the tissue. This technology can be incorporated into a handheld probe for intraoperative tissue characterization.

Within our research group, this technology has been investigated for the ability to discriminate tumor from healthy tissue in lung, liver, breast, and colorectal cancer specimens. ${ }^{2-5}$ For example, using the fat and water content obtained from the near-infrared (NIR) part of the reflectance spectrum, it was possible to distinguish tumor from healthy fat tissue in breast specimens with a sensitivity and specificity of $100 \%{ }^{4}$ Similar numbers were obtained in discriminating colorectal cancer from healthy colorectal wall (muscle) using an advanced classification algorithm on both the visual and the NIR part of the spectrum. ${ }^{5}$

DRS and related technologies have been investigated for use as noninvasive screening tools for early detection of malignancies in the oral cavity. ${ }^{6-13}$ These studies focused on the discrimination between (pre) malignant, benign, and healthy mucosa. The instruments used measured the reflected light

$1083-3668 / 2018 / \$ 25.00$ @ 2018 SPIE 

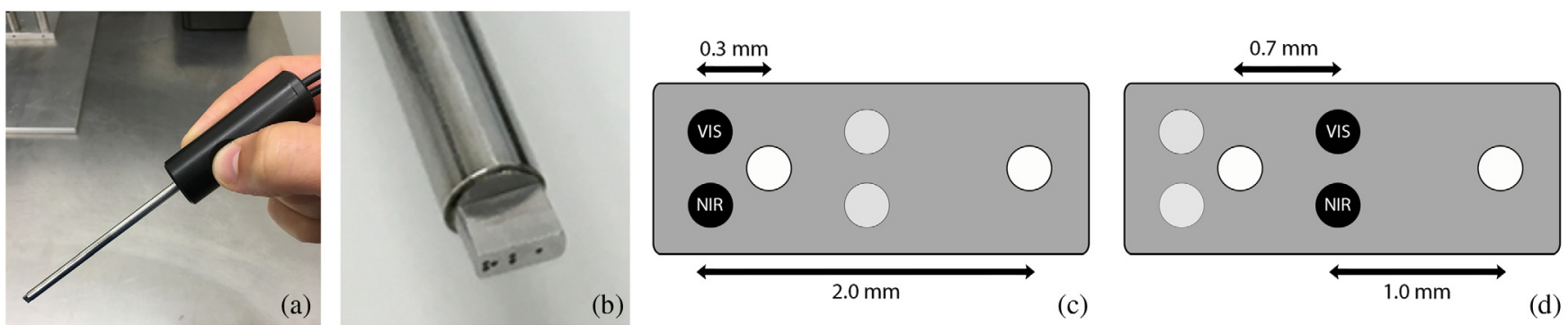

Fig. 1 (a) The handheld probe of the DRS system used, with a (b) close up of the tip of the probe. (c) and (d) Two different fiber configurations are used to obtain the diffuse reflectance spectra from four different distances between source and detection fibers.

over a wavelength range within 350 to $1000 \mathrm{~nm}$ and translated the measured spectra into tissue components. Amelink et al. ${ }^{7}$ measured a significant decrease in microvascular oxygenation and scattering amplitude and an increase in blood content and scattering slopes in tumor tissue, compared to healthy mucosa. Likewise, Stephen et al. ${ }^{12}$ showed that tumor tissue reflects more light of the two wavelengths 545 and $575 \mathrm{~nm}$, the wavelengths specifically for the absorption by oxyhemoglobin components, compared to healthy mucosa. In various studies, sensitivities of $82 \%$ to $97 \%$ and specificities of $87 \%$ to $100 \%$ have been reported for discriminating oral cavity tumor from healthy mucosa. . $^{9,11,12}$

In contrast to these studies, which concentrate on early tumor detection on the mucosal surface, we aim to use DRS as an intraoperative tool to evaluate the deep resection plane of oral cavity tumors. Hence, we want to differentiate tumor tissue from healthy muscle tissue at the resection plane of the deep resection margin. Furthermore, the main absorber in the visual wavelength range, used by the above-mentioned studies, is blood. They showed that the detection of the microvasculature played a significant role for tumor diagnosis. In contrast to those studies, we would like to use the technology intraoperatively, in an environment where blood is present, and where the blood components will not indicate the tissue type that has been measured. Thus, for intraoperative use of the DRS technology, we extended our wavelength range toward the NIR (1000 to $1700 \mathrm{~nm}$ ), in which the absorption of light by blood is negligible. ${ }^{2,4}$

With the aim to assess the deep resection margin during oral cavity cancer surgery, we evaluated whether DRS (400 to $1600 \mathrm{~nm}$ ) can discriminate tumor from healthy oral muscle tissue, in an ex-vivo setting.

\section{Materials and Methods}

\subsection{Patient Population}

Patients undergoing surgery for the removal of oral cavity cancer were considered for this study. Despite different locations of the tumors throughout the oral cavity, all measurements were performed on the same tumor type and on healthy oral muscle tissue. All patients were treated in the Netherlands Cancer Institute-Antoni van Leeuwenhoek, Amsterdam. All ethical guidelines for ex-vivo human studies were followed.

\subsection{Diffuse Reflectance Spectroscopy}

Measurements were obtained with a system consisting of a broadband tungsten halogen light for illumination and two different spectrometers to record the diffuse reflectance spectra from the tissue. The first spectrometer contained a silicon detector resolving the visual light between 400 and $1100 \mathrm{~nm}$ (Andor Technology, DU420A-BRDD), and the second spectrometer contained an InGaAs detector resolving light in the NIR region from 800 to $1700 \mathrm{~nm}$ (Andor Technology, DU492A-1.7). ${ }^{14}$ The optical fibers guiding the light from the light source toward the tissue and the light reflected from the tissue toward the spectrometers are in contact with the tissue via a handheld probe. Multiple diffuse reflectance spectra were obtained for different distances between source fiber and detector fiber: $0.3,0.7,1$, and $2 \mathrm{~mm}$ (Fig. 1). Different source-detector distances were used to obtain different sampling depths.

\subsection{Measurement Workflow}

Directly after resection, the specimen was brought to the pathology department, where the resection margins were inked according to standard pathological protocol. The pathologist localized the tumor by palpation and cut the specimen into two parts, right through the middle of the tumor. At the cut surface, suspected tumor and healthy tissue areas were pointed out by the pathologist. Based on this, the measurement locations were chosen in both tumor tissue and healthy muscle tissue. A red, green, and blue (RGB) image was taken from the cut surface for registration purposes. DRS measurements were acquired, and the probe's position for each measurement was recorded on an RGB image. The spectroscopy system could only measure on two fibers simultaneously. As a consequence, all measurements were first acquired with the 2- and $0.3-\mathrm{mm}$ fiber distance. Subsequently, to acquire the measurements for the 1- and 0.7-mm fiber distances, the probe was positioned at the same location on the basis of the RGB images. Thereafter, the specimen was brought back to the pathology department for further routine pathological processing.

\subsection{Pathology Registration}

The locations measured with the DRS probe had to be matched with the corresponding locations on the histopathology slide, to confirm the tissue type that was measured. For this, a digital scan was made of the histopathological slide taken from the cut surface, which was registered to the RGB image (Fig. 2). Due to the fact that the specimen was deformed during the histopathological processing, a nonrigid registration algorithm was used for the registration. In this algorithm, obviously matching points in both the histopathological slide and the RGB image were visually selected. On the histopathological slide, the pathologist delineated tumor and healthy muscle tissue. The measurement locations were drawn on the RGB image, based 

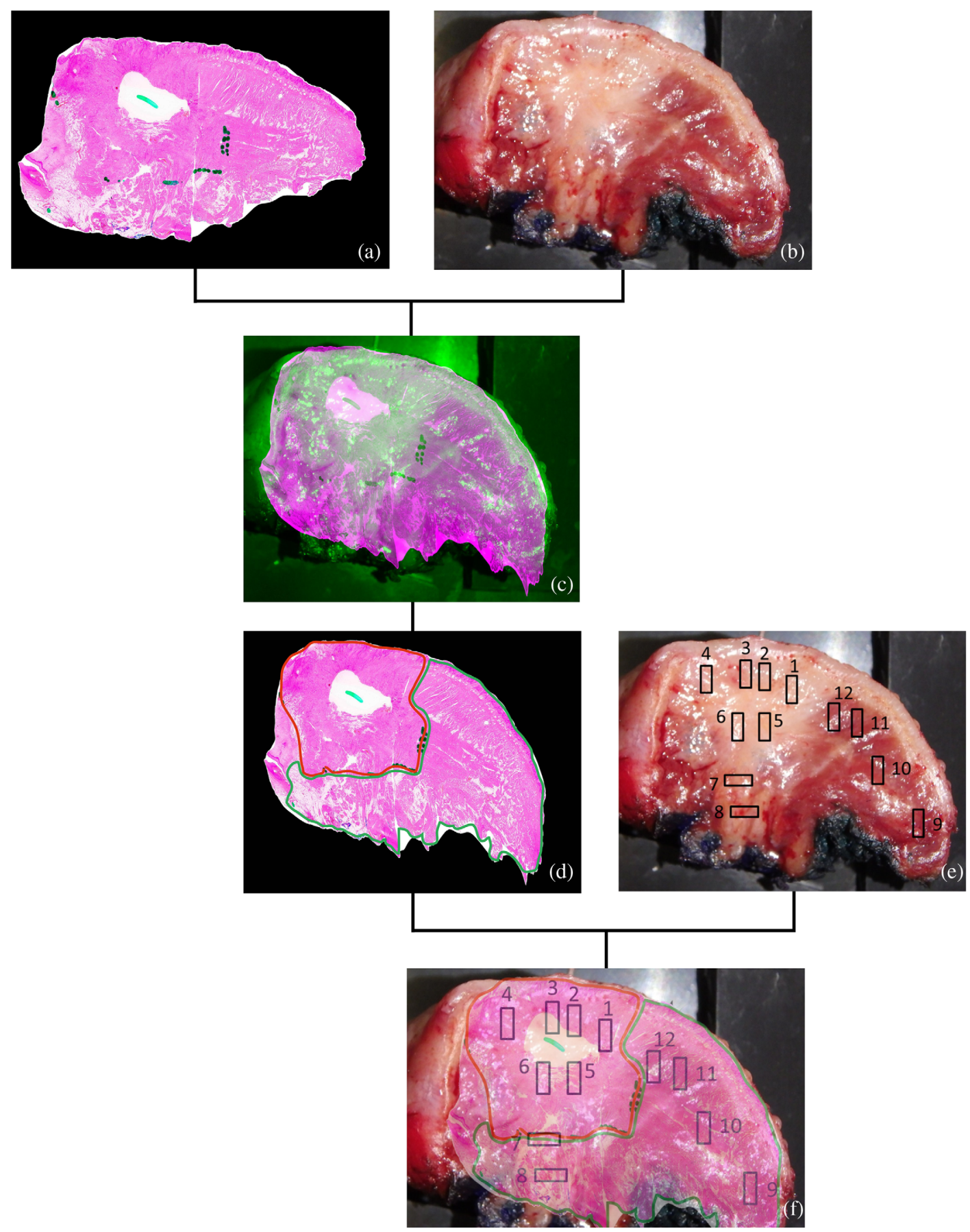

Fig. 2 Procedure for finding the histopathological classification of the locations of the DRS measurements. (a) The scanned histopathological slide is registered to (b) the RGB image. (c) The result is shown as an overlay. (d) The tumor (red) and healthy muscle (green) are then annotated on the registered histopathological slide. (e) The measurement locations are drawn onto the RGB image. (f) The registered and annotated histopathological slide is shown as an overlay on the RGB with the measurement locations.

on the images taken from the probe's position during each measurement. An overlay of the delineated registered histopathological slide on the RGB image containing the measurement locations enabled determination of the measured tissue type for each measurement. Only measurements acquired at locations with an undisputed tissue type were included in the analysis, meaning that the probe was positioned on one tissue type only and not on a boundary between two different tissue types. For algorithm development, such "pure" tissue classes are obligatory.

\subsection{Data Analysis}

The diffuse reflectance spectra were acquired from tumor and muscle measurement locations, in the wavelength range of 400 to $1600 \mathrm{~nm}$ at a $1-\mathrm{nm}$ interval. To investigate subtle changes 
in the shape of the reflectance spectra, the first ${ }^{3}$ and the secondorder derivatives were calculated with the following equations:

First-order derivative: $R^{\prime}\left(\lambda_{i}\right)=\frac{R\left(\lambda_{i+1}\right)-R\left(\lambda_{i}\right)}{\lambda_{i+1}-\lambda_{i}}$, Second-order derivative: $R^{\prime \prime}\left(\lambda_{i}\right)=\frac{R^{\prime}\left(\lambda_{i+1}\right)-R^{\prime}\left(\lambda_{i}\right)}{\lambda_{i+1}-\lambda_{i}}$,

where $\lambda_{i+1}$ and $\lambda_{i}$ are the adjacent wavelengths, and $\left(\lambda_{i}\right), R^{\prime}\left(\lambda_{i}\right)$, and $R^{\prime \prime}\left(\lambda_{i}\right)$ are the original reflectance measurements, the first-, and the second-order derivatives, respectively.

The spectral data (the first- and second-order derivatives) were downsampled with a factor 5 to reduce the number of features and prevent the algorithm from overfitting. For the classification of samples, we performed a repeated fivefold cross validation $(\mathrm{CV})$ using linear support vector machine (SVM). In this approach, patients were randomly divided into five equal sized partitions. Each partition was used as a validation set while the other four partitions were used to train the classifier.

The CV procedure was repeated 10 times, each with a different random distribution of the patients over the 5 partitions, yielding 10 tissue type predictions per measurement location. Of these predictions, mean sensitivity, specificity, accuracy (ACC), area under the curve (AUC), and Matthew's correlation coefficient (MCC) were calculated. The latter gives an indication of the quality of the classification (ranging from -1 to 1 , with -1 indicating perfect disagreement, 0 random outcome, and 1 perfect agreement between prediction and observation) and corrects for classification problems with an unequal class (tumor/healthy) size.

This analysis was repeated for each of the four data sets obtained from the different distances between source and detection fiber. Receiver-operating curve (ROC) and MCCs were used to compare results of the different fiber distances.

All data analyses were performed in MATLAB R2018a.

\section{Results}

Freshly excised specimens were measured from 28 patients with squamous cell carcinoma in the oral cavity. Nineteen of the tumors were located in the tongue (sixteen lateral tongue, two dorsal tongue, and one tongue base), one in the oropharynx, seven in the floor of the mouth (six paramedial and one anterior), and one in the left cheek. Table 1 shows the total number of spectra measured from tumor and healthy muscle tissue for each distance between source and detection fiber. In Fig. 3, the mean original reflectance spectra are shown with the accompanying standard deviations (STD) for both the tumor and healthy muscle measurement locations. Largest differences between the two tissue types are visible in the wavelength range of
600 to $1000 \mathrm{~nm}$ when measuring with $0.7,1$, or $2 \mathrm{~mm}$ between source and detection fiber. Especially, the slope of the spectra along these wavelengths differs between tumor and healthy. For the $0.3-\mathrm{mm}$ source-detector distance, there is almost a complete overlap between the STDs of both tissue types. For all source-detector distances, the NIR part of the spectrum (1000 to $1700 \mathrm{~nm}$ ) does not indicate any difference between measurements acquired on tumor or healthy tissue.

Figure 4 shows the original reflectance measurement, the slope of the original reflectance measurement (first-order derivative), and slope of the first-order derivative (the second-order derivative) for the complete spectrum and the wavelengths between 1000 and $1400 \mathrm{~nm}$. The first derivative shows differences especially around $800 \mathrm{~nm}$, indicating a large difference in slope between the original tumor and healthy reflectance spectra. Figures 4(d)-4(f) show that although the original reflectance spectrum does not necessarily indicate a difference between tumor and healthy measurements for the NIR range, both the first- and second-order derivatives do show subtle differences between the two tissue types.

Using the first- and the second-order derivatives of the spectra in a linear SVM in the 10 times fivefold $\mathrm{CV}$, MCC ranged from 0.50 to 0.71 for all fiber distances (Table 2). A 1-mm distance between source and detection fiber resulted in the highest value for the MCC (Fig. 5). Mean sensitivity, specificity, and ACC were $89 \% \pm 0.01,82 \% \pm 0.02$, and $86 \% \pm 0.01$, respectively, for this distance between source and detection fiber.

Classification outcomes for different factors for data reduction and data processing are shown in Table 3. Reducing the data with a factor 5 did not indicate a significant difference in classification outcomes; it only affected the STD. Thus, using less data, the results were more in line. This shows that, using a factor 5, the data are less subject to overfitting. Also, when comparing the different data processing methods, the classification outcomes are not significantly affected. Using the firstand second-order derivatives resulted in a minor increase in sensitivity, which is clinically most valuable. Furthermore, using the derivatives resulted in the smallest STDs.

The results obtained with a $0.7-\mathrm{mm}$ distance are comparable to the results obtained with a 1-mm distance, whereas the measurements obtained with $0.3-$ and $2-\mathrm{mm}$ distances show less accurate discrimination of tumor from healthy tissue.

\section{Discussion}

Aiming for resection margin assessment of the deep resection margin during oral cavity cancer surgery, we evaluated whether DRS (in the wavelength range of 400 to $1700 \mathrm{~nm}$ ) can accurately discriminate tumor from healthy oral muscle tissue, in an ex-vivo setting. Using a 1-mm distance between source

Table 1 Number of measurements for each distance between source and detection fiber.

\begin{tabular}{|c|c|c|c|c|}
\hline & \multicolumn{4}{|c|}{ Distance between source and detection fiber $(\mathrm{mm})$} \\
\hline & 0.3 & 0.7 & 1 & 2 \\
\hline Number of total measurements (patients) & $172(24)$ & $186(28)$ & $186(28)$ & $186(27)$ \\
\hline Number of tumor measurements & $70(20)$ & $76(23)$ & $76(23)$ & $77(22)$ \\
\hline Number of muscle measurements & $102(22)$ & $110(26)$ & $110(26)$ & $109(25)$ \\
\hline
\end{tabular}



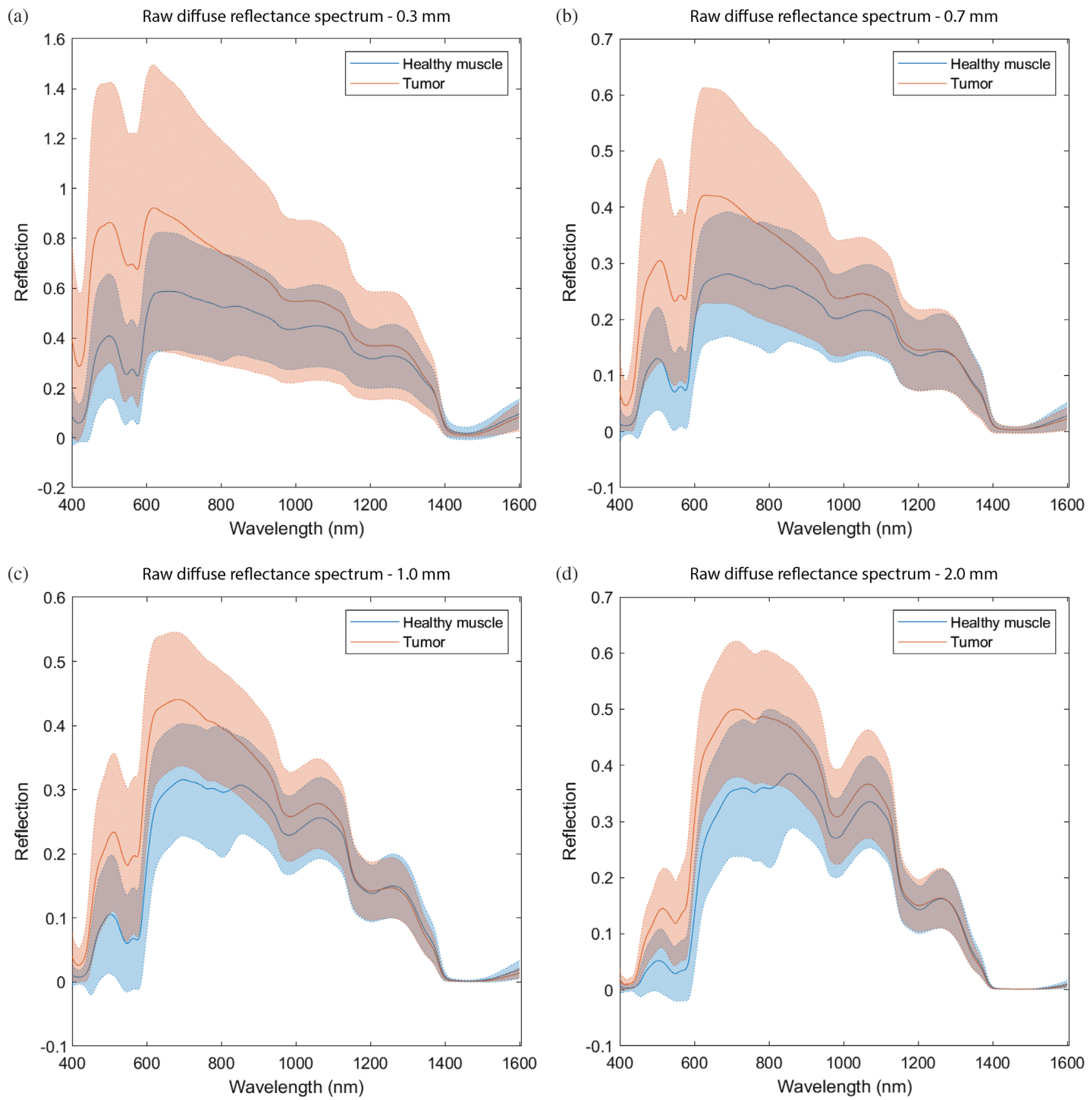

Fig. 3 Mean and STD of the raw diffuse reflectance spectra of tumor and healthy muscle for the (a) 0.3-, (b) 0.7-, (c) 1-, and (d) 2-mm distances between source and detection fiber.

and detection fiber, and a linear SVM, we found a sensitivity, specificity, and ACC of $89 \%, 82 \%$, and $86 \%$, respectively.

The sampling depth of a DRS probe is defined as the depth reached by $50 \%$ of the photons. This depth depends on different factors, such as the optical properties of the tissue, the geometry of the probe, and the wavelength of the light. ${ }^{15}$ Hennessy et al. ${ }^{15}$ used a Monte Carlo model of DRS to investigate the effect of the source-detector distance and the wavelength on the sampling depth of photons collected by a DRS probe. The source-detector distance of 0.25 and $1.0 \mathrm{~mm}$ measured sampling depths of up to 0.3 and $0.7 \mathrm{~mm}$, respectively. Thus, with a larger sourcedetector distance, larger sampling depths can be measured. They also showed that the exact sampling depth was wavelength dependent: within the visual range of 400 to $700 \mathrm{~nm}$, the sampling depth varied between 0.4 and $0.7 \mathrm{~mm}$ for a sourcedetector distance of $1.0 \mathrm{~mm}$. According to these data, we estimated that in our study a source-detector distance of $1.0 \mathrm{~mm}$ should be able to measure a sampling depth between 0.4 and $0.7 \mathrm{~mm}$, and a source-detector distance of $2 \mathrm{~mm}$ should at least be able to measure at a depth of $>1 \mathrm{~mm}$.

We looked at 0.3-, 0.7-, 1.0-, and 2-mm distances between source and detection fiber. In our study, both the $0.7-$ and $1-\mathrm{mm}$ categories were superior in discriminating tumor from healthy tissue, over the 0.3 - and 2-mm categories. This could be explained by the methodology we used to validate the data. We labeled the measurements with a tumor or healthy label 

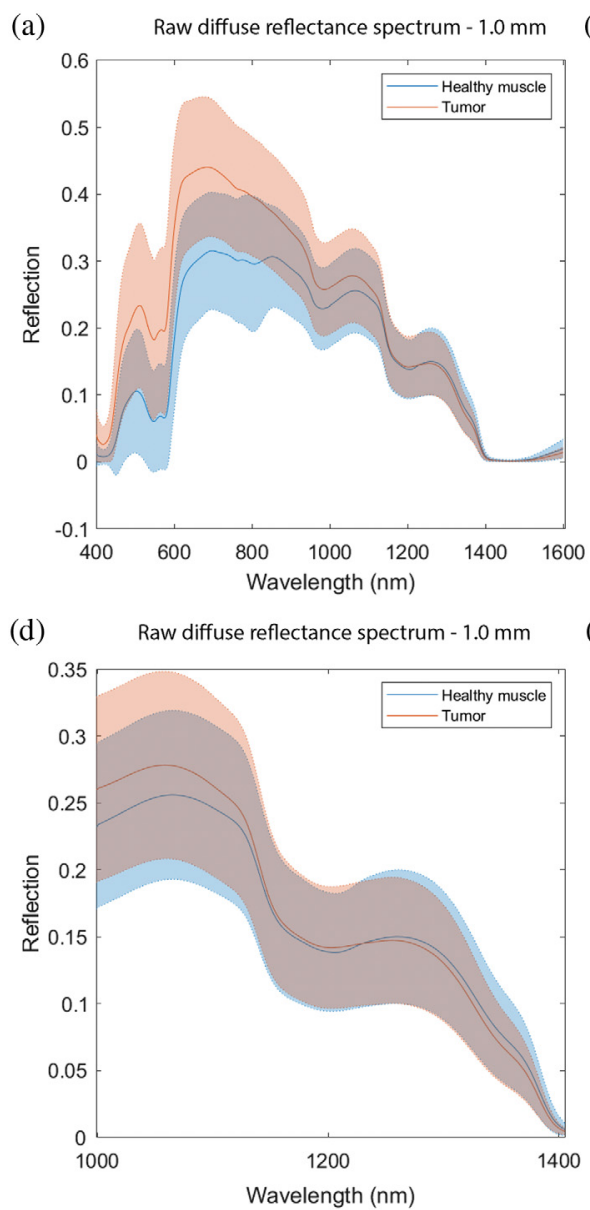

(b)

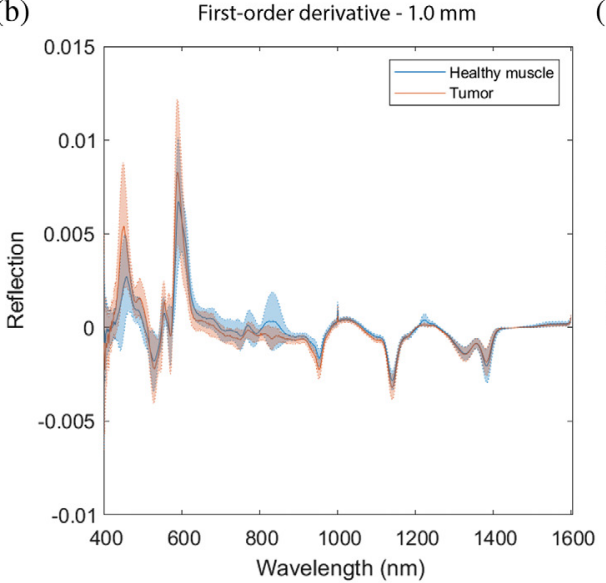

(e)

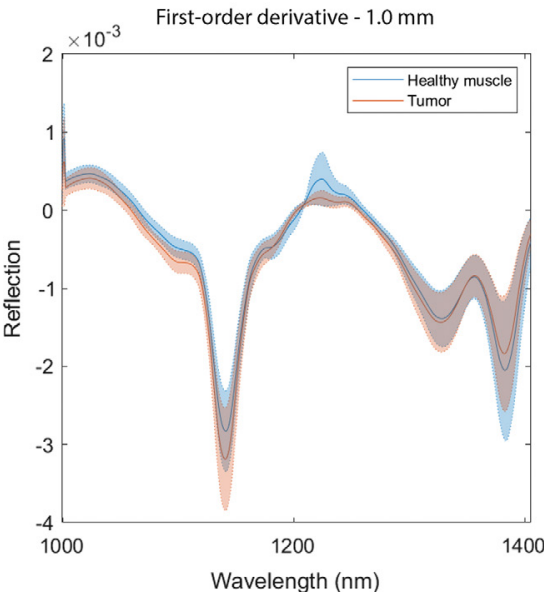

(c)

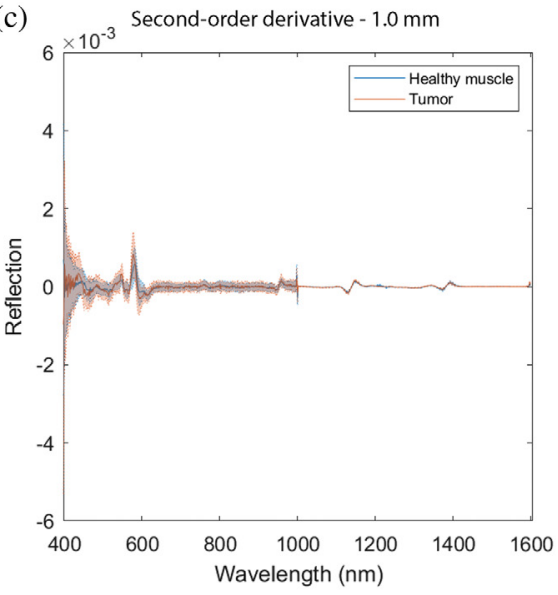

(f)

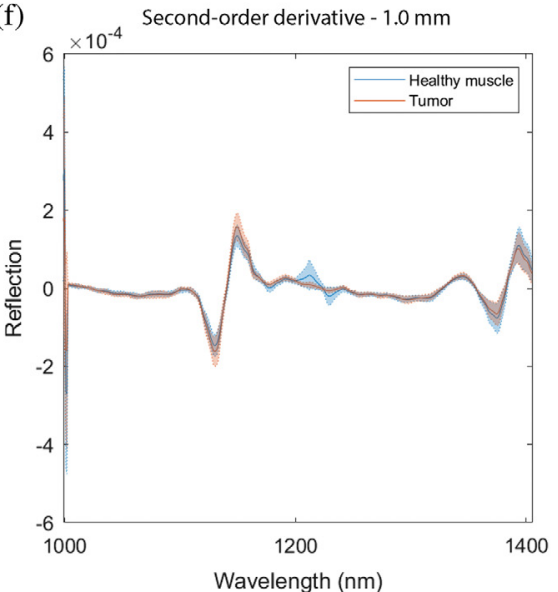

Fig. 4 Mean and STD of the diffuse reflectance spectra of tumor and healthy muscle for the 1-mm source-detector distance, plotted as (a) raw data, (b) first-order derivative, and (c) second-order derivative. (d), (e), and (f) Show the raw data, first-order derivative, and second-order derivative for the wavelength band 1000 to $1400 \mathrm{~nm}$, respectively.

Table 2 Classification results of 10 times fivefold CV with a linear SVM.

\begin{tabular}{|c|c|c|c|c|c|c|c|c|}
\hline & \multicolumn{8}{|c|}{ Distance between source and detection fiber $(\mathrm{mm})$} \\
\hline & \multicolumn{2}{|c|}{0.3} & \multicolumn{2}{|c|}{0.7} & \multicolumn{2}{|c|}{1} & \multicolumn{2}{|c|}{2} \\
\hline & Mean & STD & Mean & STD & Mean & STD & Mean & STD \\
\hline Sensitivity & 0.83 & 0.01 & 0.89 & 0.01 & 0.89 & 0.01 & 0.84 & 0.01 \\
\hline Specificity & 0.66 & 0.04 & 0.81 & 0.02 & 0.82 & 0.02 & 0.76 & 0.02 \\
\hline $\mathrm{ACC}$ & 0.76 & 0.01 & 0.86 & 0.01 & 0.86 & 0.01 & 0.81 & 0.01 \\
\hline AUC & 0.81 & 0.01 & 0.90 & 0.01 & 0.91 & 0.01 & 0.86 & 0.01 \\
\hline MCC & 0.50 & 0.03 & 0.70 & 0.02 & 0.70 & 0.02 & 0.60 & 0.02 \\
\hline
\end{tabular}

based on the first histopathological section of the complete surface of the specimen. Due to the direction of the histopathological slices and the orientation of the specimen in the paraffin block, it is not known from what depth this slice originated. It could have been that the absolute superficial layer, being measured with the 0.3 -mm source-detector distance, was not present in our histopathological section, because it was sliced off before the slice including the entire intact surface was reached in the cutting process. On the other hand, in the 2 -mm source-detector distance, the category with the largest sampling depth, it could have been the case that, besides traveling through a superficial layer of tumor tissue, the light also could have been traveling through an underlying layer of healthy tissue. Since we did not measure the tumor thickness at the measurement locations, we could not accurately validate the data for this larger sampling depth. 


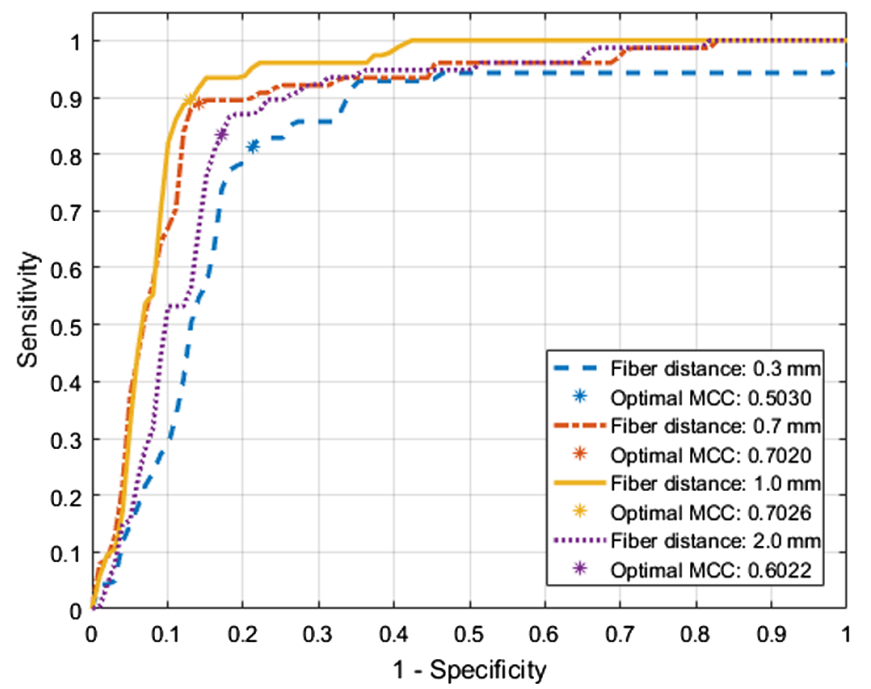

Fig. 5 ROC curves of the different distances between source and detection fiber. Cutoff points are chosen based on the maximum MCC value.

The current clinical practice guidelines in head and neck cancer define a close margin as tumor cells found within $<5 \mathrm{~mm}$ from the resection surface. However, there is no consensus with regard to whether $<5 \mathrm{~mm}$ is the right definition of "close." Alicandri-Ciufelli et al. ${ }^{16}$ reported in their systematic review that a range of 2 to $7 \mathrm{~mm}$ is considered close. Also, specifically for the tongue, Zanoni et al. ${ }^{17}$ recently suggested to redefine the definition of close margins for patients with squamous cell carcinoma. In their study, local recurrence-free survival was significantly affected only with surgical margins of $\geq 2.2 \mathrm{~mm}$. Thus, to be able to use DRS in the detection of close margins, the required sampling depth is $>2 \mathrm{~mm}$.

In our study, the discriminative power of tumor from healthy tissue was not the strongest for the largest sampling depth obtained with the 2-mm source-detector distance, due to the validation method of this category. Thus, we recommend that future work focus on improving the validation method for larger sampling depths. As an initial approach, US could be used to measure the tumor thickness at the measurement locations. Several studies have shown that the tumor thickness can be accurately measured using US. ${ }^{18,19}$

As far as we are aware, this is the first study reporting on the use of DRS in discriminating oral cavity tumor from healthy oral muscle tissue, which is essential for the intraoperative application we are aiming at. Other studies have been reporting on the use of DRS for diagnosing oral cavity cancer and are, therefore, focused on discriminating tumor from healthy mucosa, a completely different tissue type. ${ }^{6-13}$ Therefore, it is not directly possible to compare our results with these studies. At the moment, oral cavity tumor resection is performed without any intraoperative feedback on the presence of tumor cells at the tumor resection margins. So far, there are only studies reporting on the in-vivo use of US to obtain tumor-free margins in tongue surgery. ${ }^{19-21}$ In these studies, US was used to position needles as an indication for the safety margin during the resection. Also, Songra ${ }^{18}$ used a metal retractor into the surgical cut, halfway during resection to evaluate the resection margin with US. Other research on this topic focused on ex-vivo evaluation of the resection margins, e.g., by MR. Steens et al., ${ }^{22}$ reported in six out of seven patients a resection margin within a 2-mm range of the resection margin reported by histopathology. Both US and MR provide image guidance during surgery, while we aim to use DRS for tissue characterization. Therefore, DRS and US/MR are not mutually exclusive and could be used complementarily to each other.

Clinically, we would like to use DRS as an intraoperative tool to distinguish oral cavity tumor tissue from healthy muscle tissue at the resection plane. The surgeon could position the probe at a suspicious location at the deep resection margin. After measuring the tissue with DRS, the reflectance spectrum will be compared to the database that was used to train the SVM, and a prediction can be made on the type of the measured tissue, real time. A scale on a monitor will indicate the likelihood of measuring tumor tissue. Detection of tumor tissue at the deep resection margin will allow for direct re-excision and thus reduce the involved margins. Intraoperative use of DRS is feasible, because measurements do not delay the surgery and the results can be made available in real time. With the reported sensitivity and specificity in this ex-vivo study, it would be interesting to further develop the technology into a surgical instrument, which can be sterilized for in-vivo use.

Table 3 Classification results of 1-mm distance between source and detection fiber with different factors for data reduction and data processing.

\begin{tabular}{|c|c|c|c|c|c|c|c|c|c|c|c|c|c|c|}
\hline & \multicolumn{6}{|c|}{$\begin{array}{l}\text { Variable downsampling factor used with first- } \\
\text { and second-order derivatives }\end{array}$} & \multicolumn{8}{|c|}{$\begin{array}{l}\text { Variable processing of diffuse reflectance spectra used with } \\
\text { downsampling factor } 5\end{array}$} \\
\hline & \multicolumn{2}{|c|}{ None } & \multicolumn{2}{|c|}{ Factor 3} & \multicolumn{2}{|c|}{ Factor 5} & \multicolumn{2}{|c|}{ None } & \multicolumn{2}{|c|}{$\begin{array}{l}\text { First-order } \\
\text { derivative }\end{array}$} & \multicolumn{2}{|c|}{$\begin{array}{l}\text { Second-order } \\
\text { derivative }\end{array}$} & \multicolumn{2}{|c|}{$\begin{array}{l}\text { First- and } \\
\text { second-order } \\
\text { derivative }\end{array}$} \\
\hline & Mean & STD & Mean & STD & Mean & STD & Mean & STD & Mean & STD & Mean & STD & Mean & STD \\
\hline Sensitivity & 0.88 & 0.01 & 0.87 & 0.02 & 0.89 & 0.01 & 0.88 & 0.01 & 0.88 & 0.01 & 0.86 & 0.02 & 0.89 & 0.01 \\
\hline Specificity & 0.83 & 0.04 & 0.83 & 0.03 & 0.82 & 0.02 & 0.84 & 0.03 & 0.79 & 0.06 & 0.79 & 0.04 & 0.82 & 0.02 \\
\hline ACC & 0.86 & 0.02 & 0.85 & 0.02 & 0.86 & 0.01 & 0.86 & 0.01 & 0.84 & 0.02 & 0.84 & 0.01 & 0.86 & 0.01 \\
\hline AUC & 0.91 & 0.01 & 0.90 & 0.01 & 0.91 & 0.01 & 0.91 & 0.01 & 0.89 & 0.01 & 0.90 & 0.01 & 0.91 & 0.01 \\
\hline MCC & 0.71 & 0.03 & 0.70 & 0.03 & 0.70 & 0.02 & 0.72 & 0.03 & 0.67 & 0.06 & 0.67 & 0.03 & 0.70 & 0.02 \\
\hline
\end{tabular}




\section{Conclusion}

This ex-vivo study showed that DRS can discriminate tumor from healthy oral tissue. Using a linear SVM in a 10 times fivefold CV, sensitivity, specificity, and ACC measures of $89 \%$, $82 \%$, and $86 \%$ were obtained. Future work should focus on accurate validation methods for larger sampling depths to allow real-time guidance during oral cavity tumor excision.

\section{Disclosures}

This study was supported by Philips Research, Eindhoven, Netherlands. The author who is affiliated with Philips Research (BHW) has financial interests in the subject matter, materials, and equipment only in the sense that he is an employee of Philips. None of the other authors has any financial relationship with Philips Research or conflicts of interest.

\section{Acknowledgments}

We would like to thank the NKI-AvL head and neck surgeons and the pathology department for their help in the collection of the data.

\section{References}

1. R. W. Smits et al., "Resection margins in oral cancer surgery: room for improvement," Head Neck 38(Suppl. 1), E2197-E2203 (2016).

2. J. W. Spliethoff et al., "Improved identification of peripheral lung tumors by using diffuse reflectance and fluorescence spectroscopy," Lung Cancer 80(2), 165-171 (2013).

3. J. W. Spliethoff et al., "Monitoring of tumor radio frequency ablation using derivative spectroscopy," J. Biomed. Opt. 19(9), 097004 (2014).

4. L. L. de Boer et al., "Fat/water ratios measured with diffuse reflectance spectroscopy to detect breast tumor boundaries," Breast Cancer Res. Treat. 152(3), 509-518 (2015).

5. E. J. M. Baltussen et al., "Diffuse reflectance spectroscopy as a tool for real-time tissue assessment during colorectal cancer surgery," J. Biomed. Opt. 22(10), 106014 (2017).

6. C. Yu et al., "Quantitative spectroscopic imaging for noninvasive early cancer detection," Opt. Express 16(20), 16227-16239 (2008).

7. A. Amelink et al., "Non-invasive measurement of the morphology and physiology of oral mucosa by use of optical spectroscopy," Oral Oncol. 44(1), 65-71 (2008).
8. D. C. de Veld et al., "Autofluorescence and diffuse reflectance spectroscopy for oral oncology," Lasers Surg. Med. 36(5), 356-364 (2005).

9. R. A. Schwarz et al., "Noninvasive evaluation of oral lesions using depth-sensitive optical spectroscopy," Cancer 115(8), 1669-1679 (2009).

10. S. McGee et al., "Anatomy-based algorithms for detecting oral cancer using reflectance and fluorescence spectroscopy," Ann. Otol. Rhinol. Laryngol. 118(11), 817-826 (2009).

11. J. L. Jayanthi et al., "Diffuse reflectance spectroscopy: diagnostic accuracy of a non-invasive screening technique for early detection of malignant changes in the oral cavity," BMJ Open 1(1), e000071 (2011).

12. M. M. Stephen et al., "Diagnostic accuracy of diffuse reflectance imaging for early detection of pre-malignant and malignant changes in the oral cavity: a feasibility study," BMC Cancer 13, 278 (2013).

13. R. J. Mallia et al., "Diffuse reflection spectroscopy: an alternative to autofluorescence spectroscopy in tongue cancer detection," Appl. Spectrosc. 64(4), 409-418 (2010).

14. R. Nachabe et al., "Estimation of biological chromophores using diffuse optical spectroscopy: benefit of extending the UV-VIS wavelength range to include 1000 to $1600 \mathrm{~nm}, "$ Biomed. Opt. Express 1(5), 1432-1442 (2010).

15. R. Hennessy et al., "Effect of probe geometry and optical properties on the sampling depth for diffuse reflectance spectroscopy," J. Biomed. Opt. 19(10), 107002 (2014).

16. M. Alicandri-Ciufelli et al., "Surgical margins in head and neck squamous cell carcinoma: what is "close'?" Eur. Arch. Otorhinolaryngol. 270(10), 2603-2609 (2013).

17. D. K. Zanoni et al., "A proposal to redefine close surgical margins in squamous cell carcinoma of the oral tongue," JAMA Otolaryngol. Head Neck Surg. 143(6), 555-60 (2017).

18. A. K. Songra, "Observation of tumour thickness and resection margin at surgical excision of primary oral squamous cell carcinoma-assessment by ultrasound," Int. J. Oral Maxillofac. Surg. 35(4), 324-331 (2006).

19. M. Kodama et al., "Ultrasonography for intraoperative determination of tumor thickness and resection margin in tongue carcinomas," J. Oral Maxillofac. Surg. 68(8), 1746-1752 (2010).

20. M. Helbig et al., "Intraoperative B-mode endosonography of tongue carcinoma," Head Neck 23, 233-237 (2001).

21. C. H. Baek et al., "Intraoral sonography-assisted resection of T1-2 tongue cancer for adequate deep resection," Otolaryngology 139(6), 805-810 (2008).

22. S. C. A. Steens et al., "Evaluation of tongue squamous cell carcinoma resection margins using ex-vivo MR," Int. J. Comput. Assist. Radiol. Surg. 12(5), 821-828 (2017).

Biographies for the authors are not available. 